\title{
Developments in Magnetic Recording Media Technology and the Role of TEM-based Microanalysis
}

\author{
Masaaki Futamoto
}

Central Research Laboratory, Hitachi Ltd., Kokubunji, Tokyo 185-8601, Japan

The areal density of hard disk drives (HDDs) has increased approximately $10^{3}$ times in the last 20 years (FIG.1). Co-alloy materials have been used as thin film recording media. The magnetic properties have been improved by tailoring the composition and microstructure of thin films. This paper reviews the TEM-based microanalysis that played a crucial role in the research and development of thin film recording media technology.

The importance of nucleation and growth control in recording media preparation was clearly recognized by observations of plan-view and cross-sectional TEM images of thin film media in early to middle ' $80 \mathrm{~s}$. The magnetic anisotropy increased greatly by introducing a proper underlayer that fixed the orientation of magnetic crystal grains through epitaxial growth. For longitudinal magnetic recording, $\mathrm{Cr}$ and various $\mathrm{Cr}$-alloy underlayers with (100) texture were employed to align the easy magnetization axis (c-axis) of Co-alloy crystals parallel to the film plane. For perpendicular recording, nonmagnetic materials with $h c p$-structure and with (0001) orientation were developed to align the c-axis perpendicular to the film plane (FIG.2) [1.2].

With increasing the areal density, the recording layer thickness had to be decreased where more precise control of media microstructure was needed, and the microstructure could be visualized by employing high-resolution TEMs. One example is introduction of nonmagnetic Co-alloy interlayer, which improved the crystallographic quality of the initial growth region of magnetic layer (FIGs.3, 4) [3,4]. It became possible to keep high magneto-crystalline anisotropy energy of Co-alloy material for a reduced magnetic layer thickness.

Magnetic crystal grain isolation is very important to get high coercivity and low media noise properties. This can be realized by enhancing non-magnetic elements' segregation and/or precipitation at magnetic crystal grain boundaries. EELS- and nm-probe EDX-TEM have been employed since middle ' 90 s to visualize the compositional microstructure (FIG.5) [5]. High-resolution microanalysis is necessary to map the elements' distribution of recent recording media where the average crystal grain size is around10 nm and the width of nonmagnetic elements' segregation is less than $1 \mathrm{~nm}$.

Furthermore, TEM-based analysis enabled the researchers to investigate the grain diameter distribution, crystallographic defects, compositional distribution along the film growth direction, etc. The knowledge is also used to model the recording medium by which we can study the magnetic and recording characteristics of future HDDs through computer simulation.

[1] M. Futamoto, Y. Honda, H. Kakibayashi, T. Shimotsu, Y. Uesaka, Japan. J. Appl. Phys. 24 (1985)L460.

[2] M. Futamoto, Y. Honda, H. Kakibayashi, K. Yoshida, IEEE Trans. Mag. MAG-21 (1985)1426.

[3] M. Futamoto, Y. Honda, Y. Hirayama, K. Itoh, H. Ide, Y. Maruyama, IEEE Trans. Mag. 32 (1996)3789.

[4] M. Futamoto, Y. Hirayama, N. Inaba, Y. Honda, A. Kikukawa, IEICE Trans. Electron. E84-C (2001)1132.

[5] M. Futamoto et al., Tech. Rep. IEICE, MR94-81 (1995-02)53, MR95-49 (1995-11)35. 

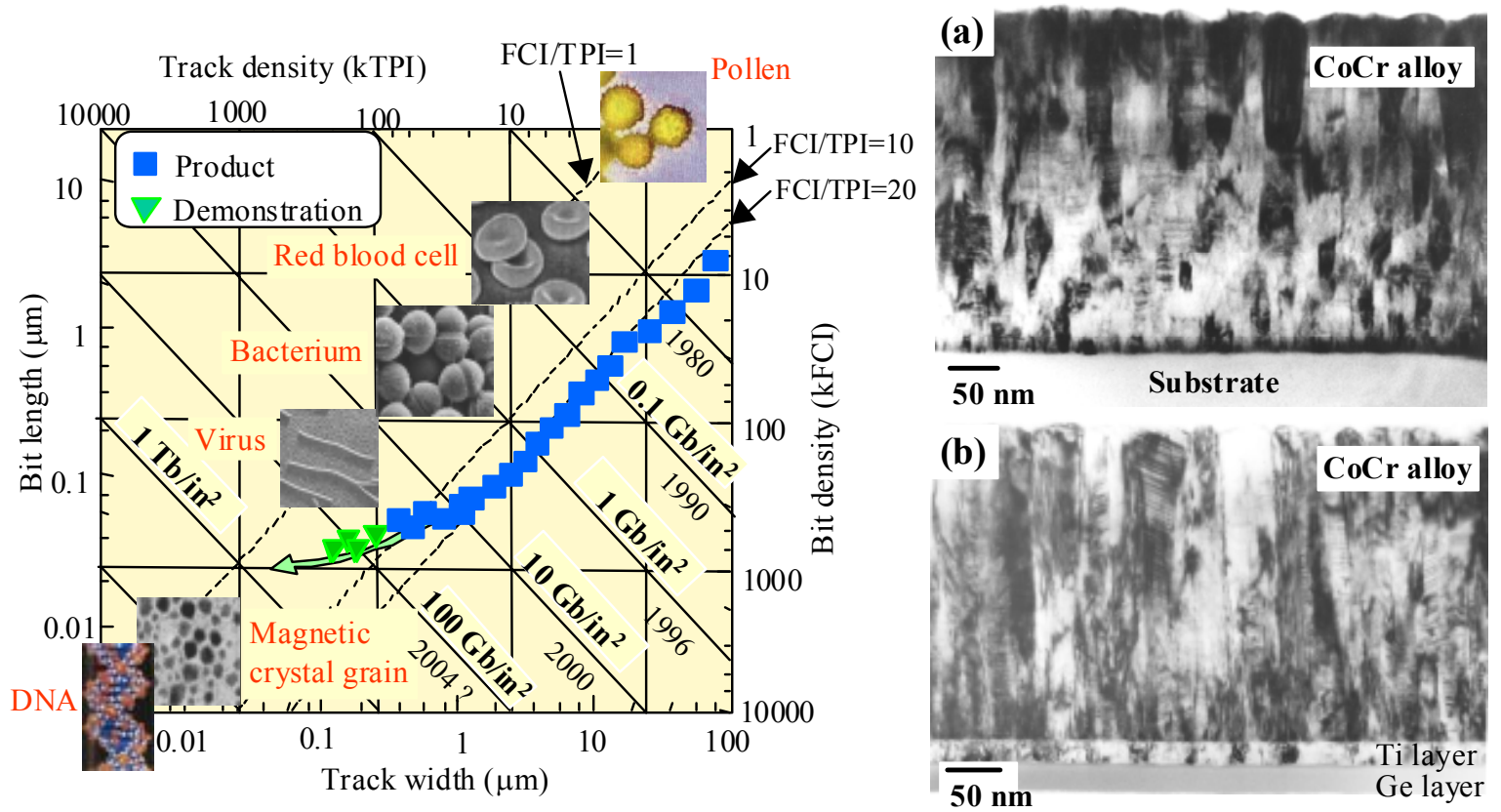

FIG.1 Decreasing trend of recording unit size in hard disk drives.

FIG.2 Cross-sectional TEM images of perpendicular recording media observed in middle ' 80 s $[1,2]$.

(a) with no-underlayer, (b) with underlayer.
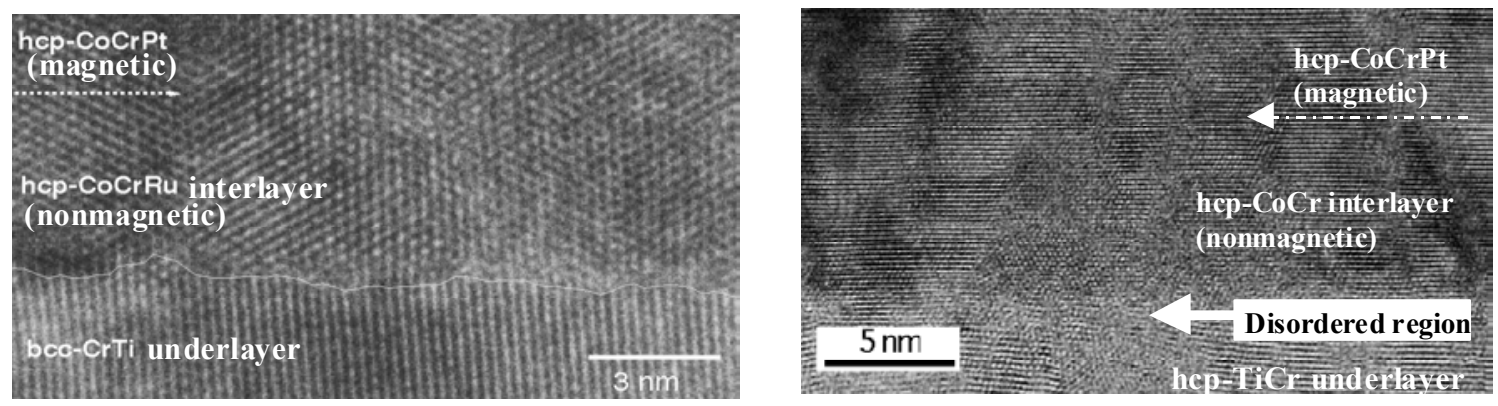

FIG.3 Cross-sectional TEM image of interface between underlayer and magnetic layer (longitudinal media) [4].

FIG.4 Cross-sectional TEM image of interface between underlayer and magnetic layer (perpendicular media) [3].
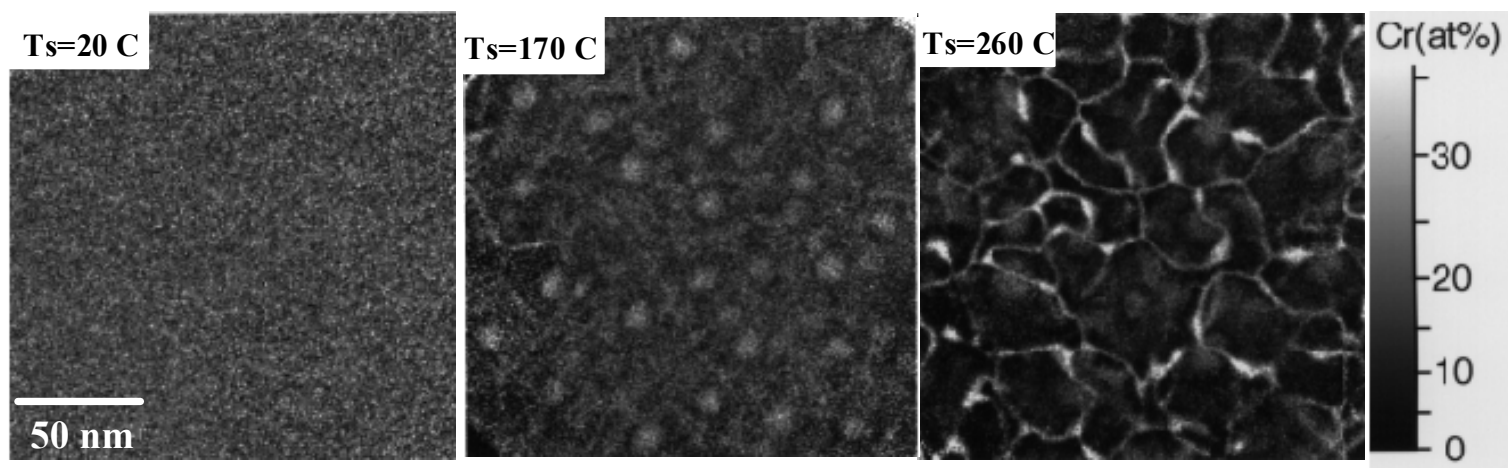

FIG.5 EELS-TEM images of CoCrTa perpendicular media [5]. Cr segregation is sensitive to substrate temperature for sputter deposition. Strong grain boundary segregation of nonmagnetic elements is necessary for low-noise and high-coercivity recording media . 